\title{
Erratum: Muir, Munroe-Chandler, and Loughead (2018)
}

In the original publication of the article Muir, I., Munroe-Chandler, K.J., \& Loughead, L. (2018), A qualitative investigation of young female dancers' use of imagery. The Sport Psychologist, 32(4), 263-274, https://doi.org/10.1123/tsp.2017-0123, the second author's name appeared incorrectly in the byline as Krista Chandler. The correct form of this author's name is Krista J. Munroe-Chandler. The online version of this article has been corrected. 